Proceedings of the ASME Turbo Expo 2019:

Turbomachinery Technical Conference \& Exposition

June 17-21, 2019, Phoenix, AZ,

GT2019-91146

\title{
SCALE-RESOLVING SIMULATIONS OF A FUNDAMENTAL TRAILING-EDGE COOLING SLOT USING A DISCONTINUOUS-GALERKIN SPECTRAL-ELEMENT METHOD
}

\author{
Anirban Garai \\ Science and Technology Corporation \\ Hampton, VA, USA
}

\author{
Scott M. Murman \\ Nateri K. Madavan \\ NASA Ames Research Center \\ Moffett Field, CA, USA
}

\begin{abstract}
The accurate prediction of turbulent mixing in high-pressure turbines that incorporate various airfoil surface-cooling strategies is becoming increasing critical to the design of modern gas turbine engines where the quest for improved efficiency is driving compressor overall pressure ratios and turbine inlet temperatures to much higher levels than ever before. In the present paper, a recently developed computational capability for accurate and efficient scale-resolving simulations of turbomachinery is extended to study the turbulent mixing mechanism of a simplified abstraction of an airfoil trailing-edge cooling slot a plane wall jet with finite lip thickness discharging into an ambient flow. The computational capability is based on an entropystable, discontinuous-Galerkin approach that extends to arbitrarily high orders of spatial and temporal accuracy. The numerical results show that the present simulations capture the trends observed in the experiments. Discrepancies between the simulations and experiments are believed to be due to differences in the inflow profiles and tunnel side-wall effects. The thick lip configuration leads to a thicker wake and higher unsteadiness in the wall jet compared to the thin lip. A detailed comparison of the turbulent flowfields is presented to highlight differences arising due to lip thickness variations.
\end{abstract}

\section{NOMENCLATURE}

$C_{p} \quad$ Specific heat capacity

$D \quad$ Dissipation

$\begin{array}{ll}\text { DoF } & \text { Degrees of freedom } \\ E & \text { Specific energy } \\ H & \text { Specific enthalpy } \\ M & \text { Mass flux variation term } \\ M a & \text { Mach number } \\ M C & \text { Mean convection term } \\ P & \text { Production term } \\ P r & \text { Prandtl number } \\ P s & \text { Pressure-strain term } \\ P t & \text { Pressure transport term } \\ R e & \text { Reynolds number } \\ R e_{\tau} & \text { Friction Reynolds number } \\ R e_{\theta} & \text { Momentum thickness Reynolds number } \\ S & \text { Source terms } \\ T & \text { Temperature } \\ T t & \text { Turbulent transport } \\ U & \text { Favre averaged streamwise velocity } \\ U_{\infty} & \text { Freestream velocity } \\ U_{c} & \text { Bulk velocity at slot exit } \\ V t & \text { Viscous transport } \\ c_{f} & \text { Skin friction coefficient } \\ c_{p} & \text { Static pressure coefficient } \\ p & \text { Pressure } \\ p t & \text { Total pressure } \\ t & \text { Time } \\ u & \text { Streamwise component of fluctuation velocity } \\ v & \text { Wall-normal component of fluctuation velocity } \\ w & \text { Spanwise component of fluctuation velocity } \\ & \end{array}$

DoF Degrees of freedom

E Specific energy

$H \quad$ Specific enthalpy

$M \quad$ Mass flux variation term

$M a \quad$ Mach number

$M C \quad$ Mean convection term

$P \quad$ Production term

Pr Prandtl number

$P s \quad$ Pressure-strain term

Pt Pressure transport term

Re Reynolds number

$R e_{\tau} \quad$ Friction Reynolds number

$\operatorname{Re}_{\theta} \quad$ Momentum thickness Reynolds number

Source terms

$T \quad$ Temperature

$T t \quad$ Turbulent transport

$U \quad$ Favre averaged streamwise velocity

$U_{\infty} \quad$ Freestream velocity

$U_{c} \quad$ Bulk velocity at slot exit

$V t \quad$ Viscous transport

$c_{f} \quad$ Skin friction coefficient

$c_{p} \quad$ Static pressure coefficient

$p \quad$ Pressure

pt Total pressure

$t \quad$ Time

$v \quad$ Wall-normal component of fluctuation velocity

w 


\section{INTRODUCTION}

The quest for higher efficiency in modern gas-turbine engines is driving compressor overall pressure ratios and turbine inlet temperatures to much higher levels than in past designs. This trend in turn results in high-pressure turbine (HPT) airfoils being subjected to much higher thermal loadings than ever before, with material limitations in the thermally critical regions of the HPT dictating the turbine operating temperature, efficiency, and durability. Much attention is focused on the trailing-edge region of HPT airfoils where efficient and innovative cooling techniques, such as pressure-side bleed slots, trailing-edge ejection, etc., are being used to alleviate the thermal loads. Accurate prediction methods that can improve our understanding of the mixing and interaction between the cooling and ambient flows are critical to the design of proper HPT trailing-edge cooling strategies.

Several experimental and numerical efforts aimed at understanding the influence of airfoil trailing-edge cooling slots on the overall flowfield have been reported in the literature over the years. Many of these efforts have dealt with a simplified abstraction of an airfoil trailing-edge cooling slot - a plane wall jet with finite lip thickness discharging into an ambient flow. Experimental investigations by Whitelaw [1], Kacker \& Whitelaw [2-5], and Martini et al. [6,7] showed that the wall-jet flow is highly unsteady, and that the cooling effectiveness strongly depends on the lip thickness and the blowing ratio. Reynolds-averaged Navier Stokes (RANS) simulations have been employed to predict the wall-jet cooling effectiveness [8-11]. Both steady and unsteady RANS simulations have shown significant differences between the predicted adiabatic wall effectiveness compared to the experimental results. More recently, scale-resolving simulations, based on techniques such as direct numerical simulation (DNS) or large eddy simulation (LES), have been applied to wall-jet simulations. DNS methods have been used to study the self-similarity behavior of the wall jet (see [12], and the references cited therein), while LES and hybrid LES/RANS techniques have been successfully employed to study trailing-edge slot cooling [13-16]. Sandberg et al. [17] have shown the potential of machine learning techniques to tune RANS models using LES results as a training dataset for wall-jet flow configurations.

In recent years, numerical methods based on DiscontinuousGalerkin (DG) discretization schemes are being used to perform
RANS, DNS, and LES simulations of turbomachinery flow configurations [18-28]. These methods offer several attractive features, including easy extension to arbitrarily high-order schemes on compact numerical stencils, applicability to different element types and mesh topologies, and computational efficiency. In our prior work, we have demonstrated the capability of an entropystable DG spectral-element method that we are developing to predict laminar separation and transition and surface roughness effects in LPT airfoils, and attached boundary-layer transition in a high-pressure turbine (HPT) airfoil cascade in the presence of inflow turbulence [25-28]. In this paper, we extend our method to perform scale-resolving simulations, i.e., wall-resolved LES, to analyze the flow in a plane wall jet with finite lip thickness discharging into an ambient flow.

The flow configuration chosen here is based on the experiments performed by Kacker and Whitelaw [5], and others, who studied a two-dimensional wall jet by varying the lip thickness and blowing ratio. One of the challenges in accurately predicting the mixing process in such flows is the proper enforcement of inflow boundary conditions for the computational domain. Previous studies by Ivanova \& Laskowski [15], and Naqavi et al. [16] imposed the experimentally-observed mean profiles with or without any turbulent perturbations. Other approaches, such as incorporating mean profiles from Reynolds-averaged NavierStokes simulations with some disturbances, or establishing conditions based on precursor scale-resolving simulations of the upstream flow, etc., have also been used in the literature. In the current work, we have assumed fully developed turbulent flow profiles that match the experimental Reynolds number and boundary-layer thickness, and have prescribed inflow conditions based on fully-developed turbulent channel flow for the wall jet and a fully-developed turbulent boundary layer for the ambient flow.

The paper is organized as follows. The numerical method is described briefly, followed by details of the flow configurations and assumptions. The turbulent inflow generation techniques are then described. Numerical results from the present simulations are presented and compared with experimental data and prior simulations, and the instantaneous behavior of the wall-jet flow is analyzed. Various aspects of the turbulent mixing mechanism are also examined by studying the Reynolds stresses and Reynolds stress budget as a function of the lip thickness and blowing ratio.

\section{NUMERICAL METHOD}

A space-time DG spectral-element method is used to solve the compressible Navier-Stokes equations. Diosady \& Murman [29] have shown that the entropy-variable formulation offers better numerical stability since it discretely satisfy the second law of thermodynamics (under exact integration). Piecewise polynomials of arbitrary order (up to $16^{\text {th }}$ order accuracy) in both spatial 
and temporal dimensions are used to take advantage of tensorproduct bases. The inviscid and viscous fluxes are computed using the entropy-stable approach of Ismail and Roe [30] and an interior penalty method where the penalty parameter is consistent with the second method of Bassi and Rebay [31], respectively. Twice as many quadrature points as solution points in the temporal and spatial directions are used to minimize quadrature rules. A preconditioned Jacobian-free approximate NewtonKrylov solver is used to solve the nonlinear system [32]. Further details regarding the space-time DG method can be found elsewhere (see [33], and the references cited therein). Note also that no subgrid-scale or wall models are used in the present simulations.

In order to minimize spurious reflections from the farfield and outflow computational boundaries we have implemented the perfectly matched layer (PML) technique [34] in the numerical method. We have demonstrated in prior work $[26,35]$ that the PML technique for boundary specification leads to superior results for a variety of flow configurations including low-pressure and high-pressure turbine cascades.

\section{PROBLEM SETUP}

The flow configuration is based on the experiments performed by Kacker \& Whitelaw [5] who studied the flow characteristics for a two-dimensional wall jet for different lip thicknesses and blowing ratios. In the present study, we consider a blowing ratio of 0.75 for two lip thickness values - one representing a "thick" lip (1.14yc , referred to as Cases Ac and A) and another representing a "thin" lip $\left(0.126 y_{c}\right.$, referred to as Case B). The jet Reynolds number is chosen as $\frac{U_{c} y_{c}}{v}=6.65 \times 10^{3}$, as reported in Ref. [1]. The boundary layer thickness for the external flow is obtained from the experimental observation at the slot exit. Note that the Case B configuration has also been studied by Naqavi et al. [16].

In the experiments [1], a two-dimensional, low-turbulence intensity wind tunnel with secondary air blown parallel to the base-plate of the tunnel was used. In our simulations, we have avoided the geometrical complexities upstream of the slot exit and have also chosen to ignore tunnel side-wall effects. Since the upstream flow profiles were not documented in the experiments, we have assumed a fully-developed turbulent zeropressure-gradient boundary layer for the external flow, and fully-developed turbulent channel flow for the jet.

Figure 1 represents the computational domain used in the simulations. We have utilized the multi-physics capability of the DG solver [33] to set up the problem. The computational domain consists of a turbulent boundary-layer inflow generation region, a turbulent channel inflow generation region, and PML regions at the outflow and farfield boundaries of the main computational region. The inflow generating regions are one-way coupled, while the PML regions are fully coupled with the main computational

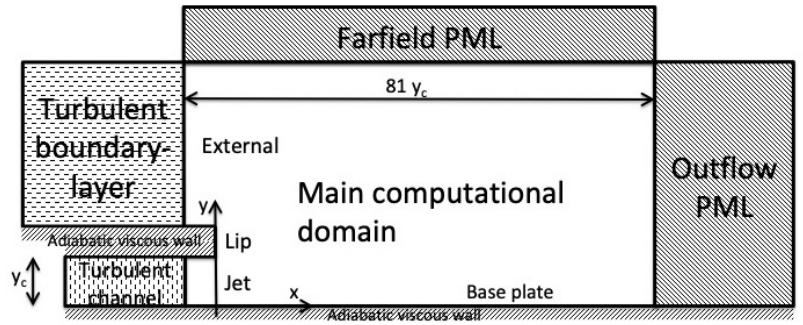

(a)

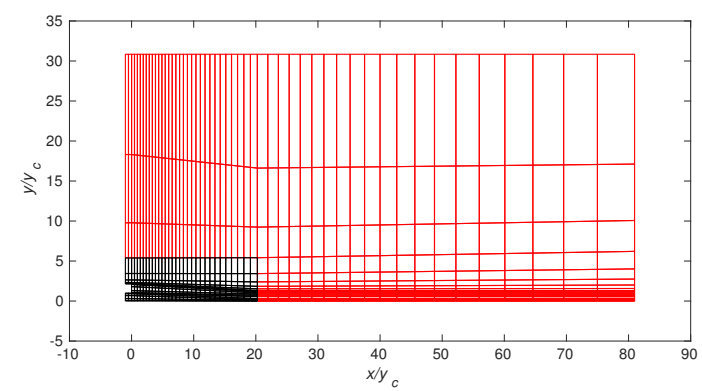

(b)

FIGURE 1: Schematic of the (a) computational domain, and (b the representative elements used in the main computational domain. Black and red colors represent $16^{\text {th }}$ and $8^{\text {th }}$ order solution regions used in the Case A simulations, respectively.

region through the boundaries. The origin of the coordinate system is established at the base plate in line with the lip of the wall jet, as shown in Fig. 1a. Adiabatic viscous wall boundary conditions are used at the base plate and the lip. The spanwise extent of the computational domain is $6 y_{c}$, similar to the values used by Naqavi et al. [16], and periodic boundary conditions are enforced in the spanwise direction. The spanwise extent is discretized using 10 elements. In the main computational domain, a refined mesh with smaller and higher-order solution elements is used in the regions $x<20 y_{c}$ and $y<5 y_{c}$ compared to the rest of the domain (Fig. 1b). Uniform $8^{\text {th }}$ order elements in the spatial directions are used for Cases Ac and B, while hybrid $16^{\text {th }}$ order elements in $x<20 y_{c}$ and $y<5 y_{c}$, and $8^{\text {th }}$ order elements in the rest of the region are used for Case A. Table 1 summarizes the number of elements in the streamwise and wall-normal directions, and the resulting number of degrees of freedom for the different flow configurations.

\section{INFLOW GENERATION}

As mentioned earlier, we have assumed a fully developed, turbulent, zero-pressure-gradient boundary layer for the external flow, and a turbulent channel profile for the jet. These fully developed turbulent profiles are generated in a streamwise and span- 
TABLE 1: Summary of the grids used in the main computational domain for the different flow configurations.

\begin{tabular}{c|c|c|c|c|c|c|c|c}
\hline Case & lip & blowing & \multicolumn{2}{|c|}{ streamwise elements } & \multicolumn{2}{|c|}{ wall-normal elements } & DoF \\
\cline { 4 - 7 } & thickness $\left(y_{c}\right)$ & ratio $\left(\frac{U_{c}}{U_{\infty}}\right)$ & $x / y_{c}<20$ & $20<x / y_{c}<80$ & jet & lip & external & \\
$\left(\times 10^{6}\right)$
\end{tabular}

wise periodic box by incorporating appropriate source terms.

The wall-normal and spanwise mesh resolutions in the boundary layer and channel regions match the resolutions in the main computational region immediately downstream shown in Fig. 1a. In order to keep the computations from becoming prohibitively expensive, coarser mesh resolutions than typically recommended for DNS computations are used in these regions, as shown in Table 2 and 3.

\section{Turbulent Boundary Layer Profiles}

To generate a turbulent zero-pressure-gradient boundary layer profile in a streamwise and spanwise periodic box, appropriate source terms are added in the mass, momentum and energy equations. The compressible Navier-Stokes equations with the source terms can be written as:

$$
\begin{gathered}
\frac{\partial \rho}{\partial t}+\frac{\partial}{\partial x_{j}}\left(\rho u_{j}\right)=S_{\rho}, \\
\frac{\partial\left(\rho u_{i}\right)}{\partial t}+\frac{\partial}{\partial x_{j}}\left(u_{j} \rho u_{i}\right)=-\frac{\partial p}{\partial x_{i}}+\frac{\partial \sigma_{i j}}{\partial x_{j}}+S_{\rho u_{i}}, \\
\frac{\partial(\rho E)}{\partial t}+\frac{\partial}{\partial x_{j}}\left(u_{j} \rho H\right)=\frac{\partial}{\partial x_{j}}\left(u_{i} \sigma_{i j}+\frac{C_{p} \mu}{\operatorname{Pr}} \frac{\partial T}{\partial x_{j}}\right)+S_{\rho E}
\end{gathered}
$$

For a zero-pressure-gradient boundary layer, $\frac{\partial \bar{p}}{\partial x}=0$. To account for the streamwise growth of the boundary layer in a streamwise periodic box, the terms corresponding to the streamwise mean gradients are added as source terms to Eqn. 1. The streamwise mean gradient terms are estimated using the turbulent boundary layer log-law profile with Cole's wake profile [36]. The streamwise mean gradients of the turbulent fluctuation quantities are assumed to be negligibly small. To generate turbulence, the flow in the streamwise and spanwise periodic box is initialized with some perturbations that are then evolved in time to reach a stationary state. Flow stationarity is monitored using the temporal evolution of the wall friction and is achieved after about $50-60$ flow-through times (based on streamwise boundary-layer domain length and $U_{\infty}$ ). The turbulent boundary layer momentum thickness in the streamwise and spanwise periodic box is calibrated by using zero-pressure-gradient turbulent boundary layer evolution theory [37] to match the experimentally observed boundary layer thickness at the slot exit.
TABLE 2: Summary of the grid characteristics in the turbulent boundary layer for the different flow configurations.

\begin{tabular}{c|c|c|c}
\hline Case & $\begin{array}{c}\text { DoF } \\
(x \times y \times z)\end{array}$ & $x^{+}$ & $z^{+}$ \\
\hline Ac & $240 \times 64 \times 80$ & 45 & 30 \\
A & $480 \times 104 \times 160$ & 22.5 & 15 \\
B & $360 \times 64 \times 80$ & 25 & 30 \\
\hline
\end{tabular}

Table 2 summarizes the streamwise and spanwise resolutions used to generate the turbulent boundary layer profiles for the different flow configurations. At the wall, the effective $y^{+}$ (wall normal cell size divided by solution order) is about 2.5 for the Cases Ac and B; and about 1 for Case A. Since we are using a compressible flow solver in the present study, we have decomposed $u=U+u^{\prime \prime}$, where $u$ represents the instantaneous velocity, $U$ the Favre-averaged velocity, and $u^{\prime \prime}$ the Favre fluctuations. Since the freestream $M a=0.1$ for the present simulations is quite low, the Reynolds- and Favre-averaged quantities are similar.

Figure 2a shows the comparison of the mean streamwise velocity with the DNS results of Spalart [38] corresponding to $R e_{\theta}=1410$. The log-law behavior is captured satisfactorily but the profiles deviate in the wake region due to the insufficient resolution at the edge of the boundary layer. Figure $2 \mathrm{~b}$ shows that the turbulent intensities are also in good agreement with Spalart's DNS results. The peak streamwise and spanwise turbulent intensities are higher compared to Spalart's results due to the larger values of $x^{+}$and $z^{+}$used here. The refined mesh simulations using $16^{\text {th }}$ order solution elements in the region $y<5 y_{c}$ for Case A move the peak turbulent intensities closer to Spalart's results compared to the other configurations, and also reduces the discontinuities at the element boundaries. Also, note the our results show higher turbulent intensities in the vicinity of the edge of the boundary layer due to insufficient mesh resolution. These spatiotemporal boundary layer profiles are used as inflow boundary conditions for the wall-jet simulations.

\section{Turbulent Channel Profiles}

The turbulent channel profiles are generated in a streamwise and spanwise periodic box by adding a source term, $\frac{2 \tau_{w}}{y_{c}}$, 


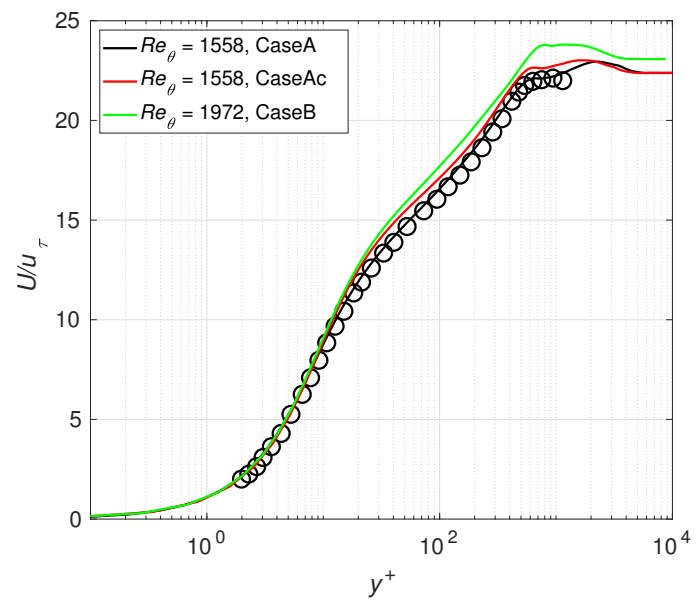

(a)

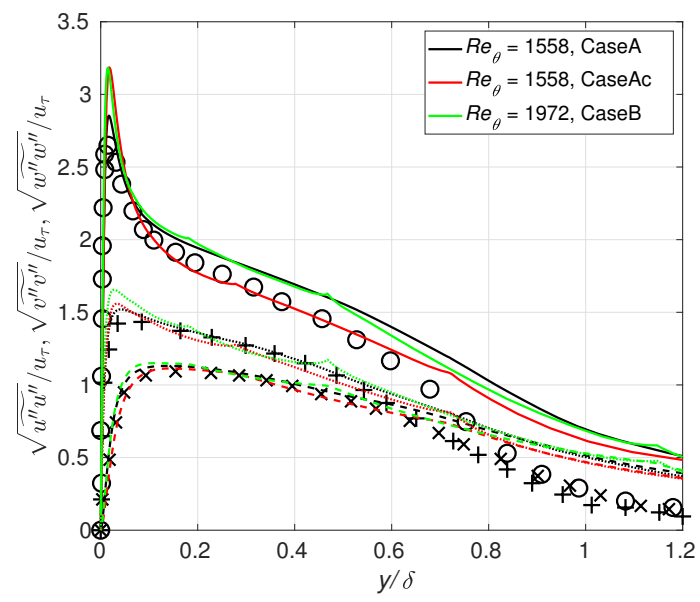

(b)

FIGURE 2: Profiles of (a) mean velocity and (b) turbulence intensities in the turbulent boundary layer for different $R e_{\theta}$. Symbols in the figures represent the DNS results of Spalart [38] corresponding to $R e_{\theta}=1410$. In Fig. b, the solid lines (circle symbols), dotted lines (plus symbols), and dashed lines (cross symbols) represent the streamwise, spanwise, and wall-normal components of the turbulent intensity, respectively.

TABLE 3: Summary of the grid characteristics of the turbulent channel used for the different flow configurations.

\begin{tabular}{c|c|c|c}
\hline Case & $\begin{array}{c}\text { DoF } \\
(x \times y \times z)\end{array}$ & $x^{+}$ & $z^{+}$ \\
\hline Ac & $48 \times 40 \times 80$ & 50 & 30 \\
A & $96 \times 80 \times 160$ & 25 & 15 \\
B & $64 \times 64 \times 80$ & 35 & 30 \\
\hline
\end{tabular}

to the $x$-momentum equation (Eqn. 1) only. The wall friction, $\tau_{w}$, is estimated using the bulk Reynolds number [36] from the experimental observations. In a manner similar to the process described above for generating the turbulent boundary layer profiles, the flow in the streamwise and spanwise periodic box is initiated with some perturbations to generate turbulence, and the simulations are then evolved to reach a stationary state. Flow stationarity is monitored using the temporal evolution of the wall friction and is typically achieved after about 100 flow-through times (based on streamwise channel domain length and $U_{\infty}$ ) .

Table 3 summarizes the streamwise and spanwise resolutions used to generate turbulent channel flow for the different flow configurations. At both walls, the effective $y^{+}$is about 2 for Cases Ac and B, and about 1 for Case A.

Figure 3 a shows that the mean streamwise velocity from the present simulations compare well with the DNS results of Moser et al. [39]. The turbulence intensity components also compare reasonably well with the DNS results (Fig. 3b). Similar to the turbulent boundary layer results, the peak $u^{+}$and $w^{+}$are overpredicted in the present simulations due to the larger $x^{+}$and $z^{+}$ values used compared to the DNS. Results for the refined mesh Case A simulations are closer to the DNS data than the coarse mesh simulations. These spatio-temporal channel profiles are used as inflow conditions for the jet.

\section{NUMERICAL RESULTS}

Flow stationarity is monitored using the temporal evolution of the wall surface pressure and skin friction and is assumed to be achieved when the temporal variation of these quantities is less that $5 \%$. Mean flow statistics are then computed by averaging both in the span and time over an additional $2-3 \tau$ $\left(\tau=80 y_{c} / U_{\infty}\right)$. A time step of $5 \times 10^{-4} \tau$ is used in the present simulations.

\section{Mesh Convergence Studies}

We have performed mesh convergence studies (Fig. 4) for the configuration with lip thickness of $1.14 y_{c}$ and a blowing ratio of 0.75 by studying the mean and turbulent velocity profiles at different streamwise locations using two different meshes, Case A and Case Ac, as described in Table 1. Similar inflow turbulent profiles are used for both cases (Figs. 2, 3). Since we are primarily interested in the flowfield characteristics for $x<20 y_{c}$ and $y<5 y_{c}$, the mesh is refined using smaller element sizes and higher solution order only in this region (see Table 1 and Fig. 1b). Figure 4 shows that the grid effects on the mean and turbulent 


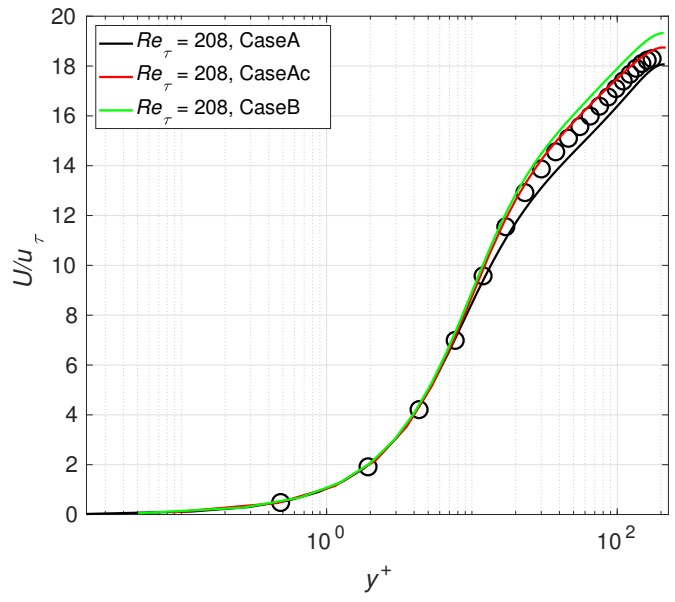

(a)

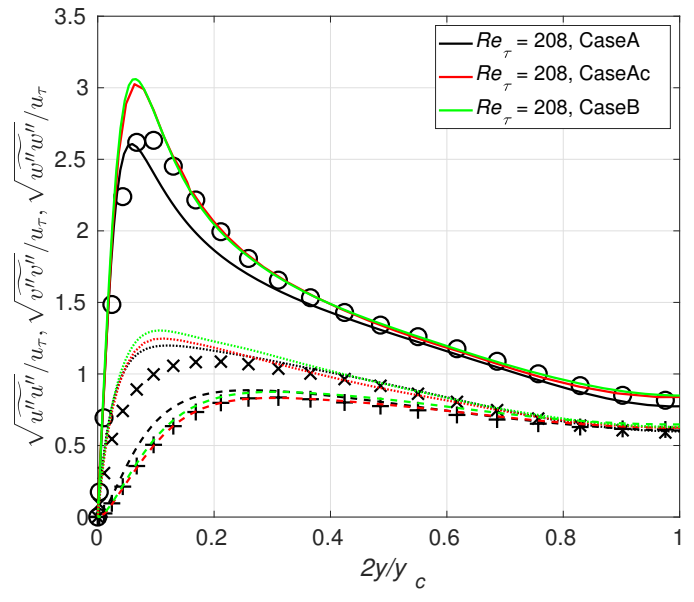

(b)

FIGURE 3: Profiles of (a) mean velocity and (b) turbulence intensities of the turbulent channel for different $R e_{\tau}$. Symbols in the figures represent the DNS results of Moser et al. [39] for $R e_{\tau}=180$ (black symbols). In Fig. b, the solid lines (circle symbols), dotted lines (plus symbols), and dashed lines (cross symbols) represent the streamwise, spanwise, and wall normal components of the turbulence intensity, respectively.

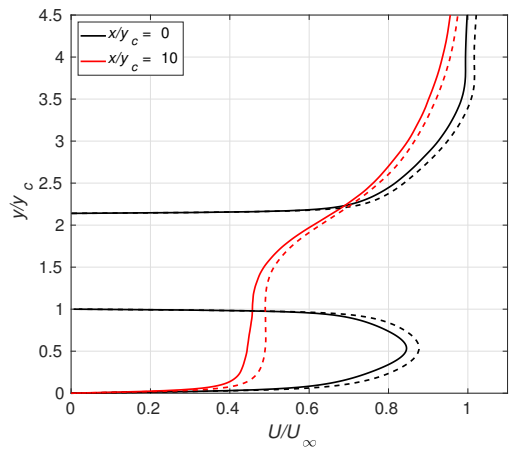

(a)

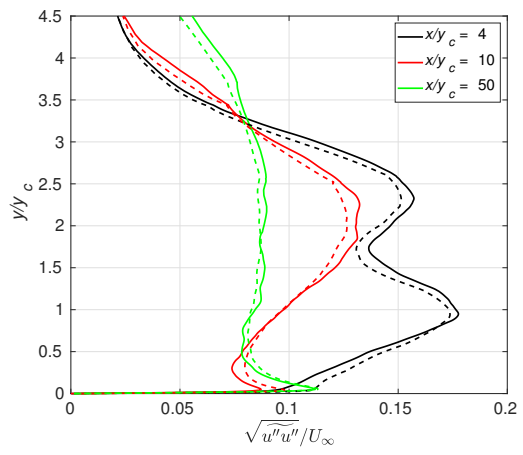

(b)

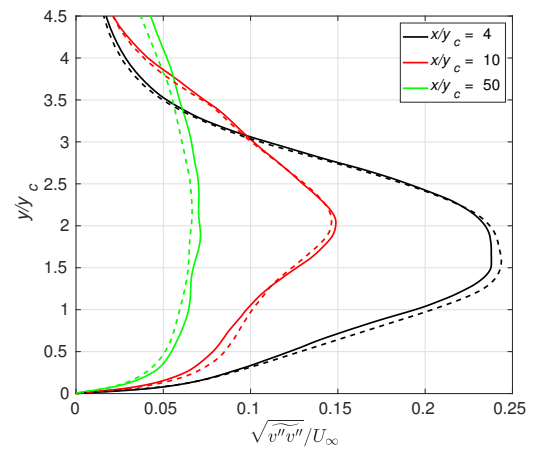

(c)

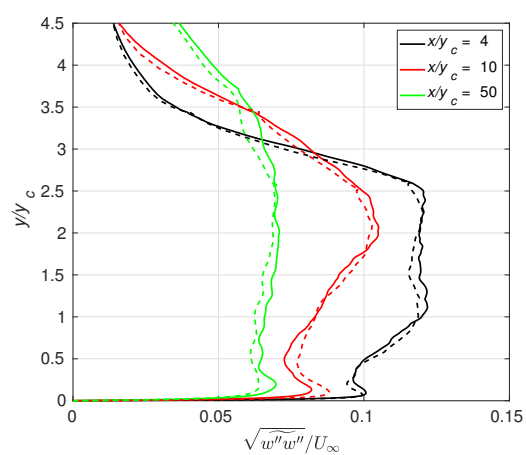

(d)

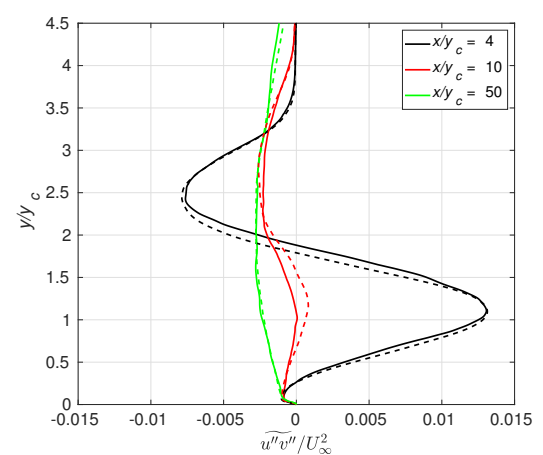

(e)

FIGURE 4: Comparison of mean and turbulent velocity profiles for Case A (solid line) and Case Ac (dashed line). 
velocity profiles are minimal. Figure 4 also shows that the discontinuities at the element boundaries decreases with the mesh refinement. The base-plate surface pressure and wall shear stress remain marginally sensitive to the mesh resolution. For the remainder of the paper, we will only present results from the Case A mesh for the configuration with lip thickness of $1.14 y_{c}$ and blowing ratio of 0.75 .

\section{Instantaneous Flow Visualization}

The instantaneous flowfields for the different configurations are studied by visualizations of the spanwise vorticity (Fig. 5) and temperature (Fig. 6) in a $x-y$ plane. Shear layers from the external boundary layer and the upper wall of the jet form two unsteady recirculation bubbles behind the lip for both Case A (Fig. 5a) and Case B (Fig. 5b). These two shear layers roll up to form a Karman vortex street. For the thicker lip (Case A), these vortices grow in size rapidly as they merge with each other, and the growth is hindered by the presence of the wall downstream. This rapid growth results in strong unsteadiness in the core of the wall jet, and causes rapid mixing between the boundary-layer and wall-jet flows (Figs. 5a, 6a). For Case B, the growth of the vortices is smaller compared to Case A due to the smaller lip thickness and the wall-jet core persists further downstream. This results in less mixing between the jet and the external boundary layer close to the lip (Figs. 5b, 6b). Similar turbulent coherent structures have also been reported in the literature $[15,16]$.

Note that $16^{\text {th }}$ order solution elements are used for Case A compared to $8^{\text {th }}$ order for Case B. As a result, Figs. 5a and 6 a show considerably more small-scale structures compared to Figs. 5b and 6b. Note also that the DG discretization inherently leads to discontinuities at the element boundaries, and the extent of these discontinuities depends on the resolution used. The $16^{\text {th }}$ order solution used for Case A has considerably less discontinuities compared to the $8^{\text {th }}$ order for Case B. In order to eliminate the discontinuities completely, one would have to perform simulations using "DNS quality" meshes that are much finer than those used here. This obviously can be done, but would be prohibitively expensive. We are currently developing a subgrid scale model [40] to address the discontinuities in the LES simulations, and are also incorporating mesh refinement approaches based on adjoint error estimation and metric-aligned meshing [41] that make more optimal use of computational resources than the uniform mesh refinement used here.

\section{Mean Velocity Profiles}

We next compare the mean streamwise velocity profiles with the experimental observations (Fig. 7). Good qualitative agreement with the experimental observations is noted for both Case $A$ and Case B. Note that we have not included any upstream or wind-tunnel side-wall geometries in the present simulations in order to keep computational costs reasonable. Also, the up- stream velocity and turbulent intensity profiles were not reported in the experiment and we have assumed fully-developed boundary layer and channel profiles in our simulations.

For Case A, the mean velocity profile at the slot exit becomes asymmetric although the inflow profiles are symmetric about the slot mid-height and match the experiment (Fig 7a). A slight underprediction in the peak jet velocity and overprediction in the outer boundary layer profile are noted at the slot exit. These are most likely due to the differences in the upstream velocity profiles. Comparisons of the mean flow suggest that these fully-developed velocity profiles are a reasonable assumption. For Case A (Fig. 7a), the mean velocity remains almost constant over the jet, and then increases to the external flow velocity at the $x / y_{c}=10$ location due to mixing of the wall jet and external flow, as seen in Figs. 5a, 6a.

For Case B, although the jet inflow profile is symmetric about the slot mid-height, the velocity profile becomes asymmetric at the slot exit, similar to the experimental observations (Fig 7b). The mean streamwise velocity profile appears to be more flat in the experiment compared to the present simulation at the slot mid-height. The external boundary layer profile agrees well with the experimental observation. As for Case A, at $x / y_{c}=10$ the mean streamwise velocity remains almost constant over the jet and then increases to the external flow velocity due to mixing of the wall jet and external flow, as seen in Figs. 5b, 6b. Similar observations have also been made by others [16].

\section{Static Pressure Distribution}

The mean static pressure distributions $\left(c_{p}=\frac{p_{s}-p_{a t m}}{p_{o}-p_{\text {atm }}}\right)$ at the base-plate for the different flow configurations are then compared with the experimental observations in Fig. 8. The numerical simulations capture the experimentally observed trends reasonably well, although the static pressure estimates from the present simulations are slightly lower. The static pressure increases rapidly from the $x=0$ location to a peak value and then decreases downstream for both Case A and Case B. The increase in the static pressure at $x<5 y_{c}$ is greater with the thicker lip.

\section{Wall Shear Stress}

The predicted base-plate wall shear stress profiles are compared with the experimental observations in Fig. 9. Although the experimentally observed trends are captured well, the present simulations underpredict the shear stress at most locations, which is consistent with the differences noted in the mean velocity profiles (Figs. 7). For Case A, the wall shear stress drops sharply from $x=0$, increases until $x \approx 6 y_{c}$, then decreases again, and gradually increases beyond $x>20 y_{c}$. This is due to the large vortex shedding from the thick lip (Fig. 5a). However, for Case $\mathrm{B}$, the wall shear stress increases from the slot exit till $x \approx 5 y_{c}$, and then gradually decreases to a constant value downstream. The observed drop in the wall shear stress at $20 y_{c}<x<30 y_{c}$ for 

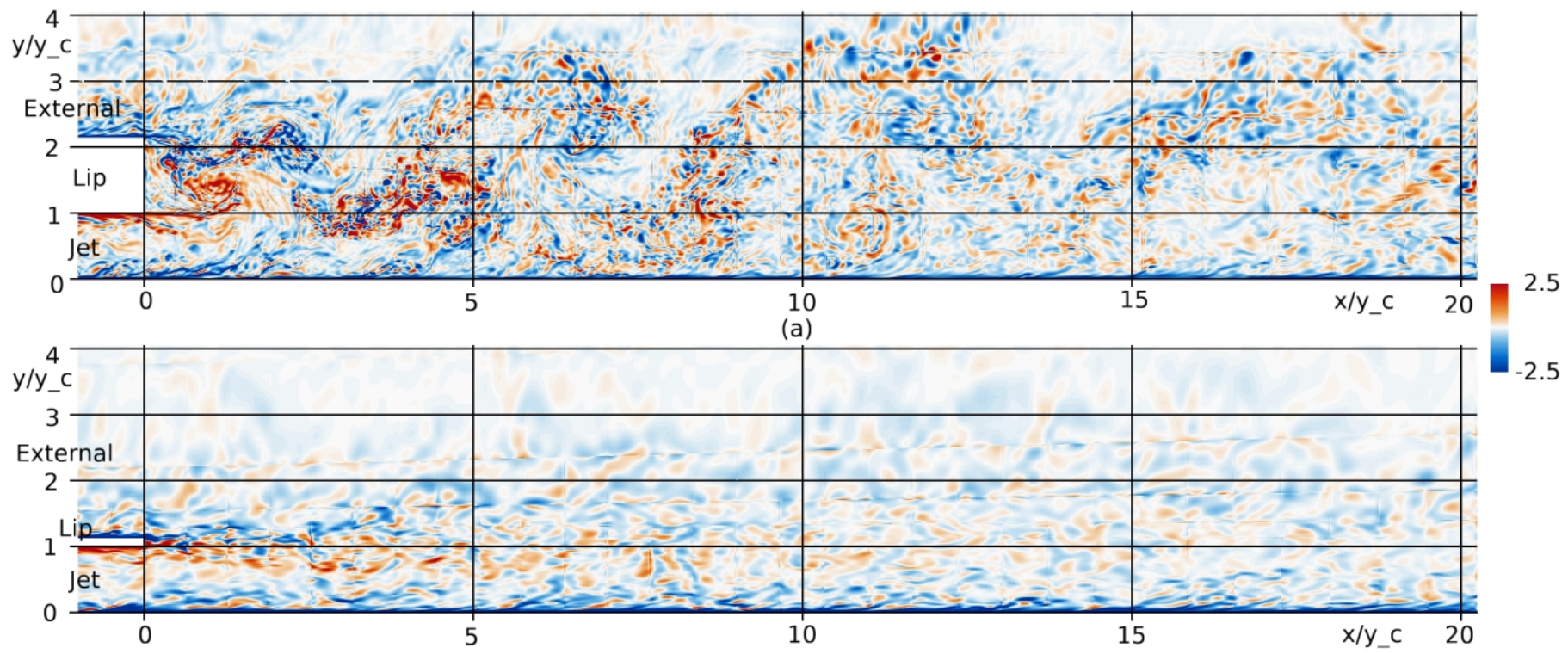

(b)

FIGURE 5: Instantaneous spanwise vorticity contours in the $x-y$ plane for (a) Case A and (b) Case B.

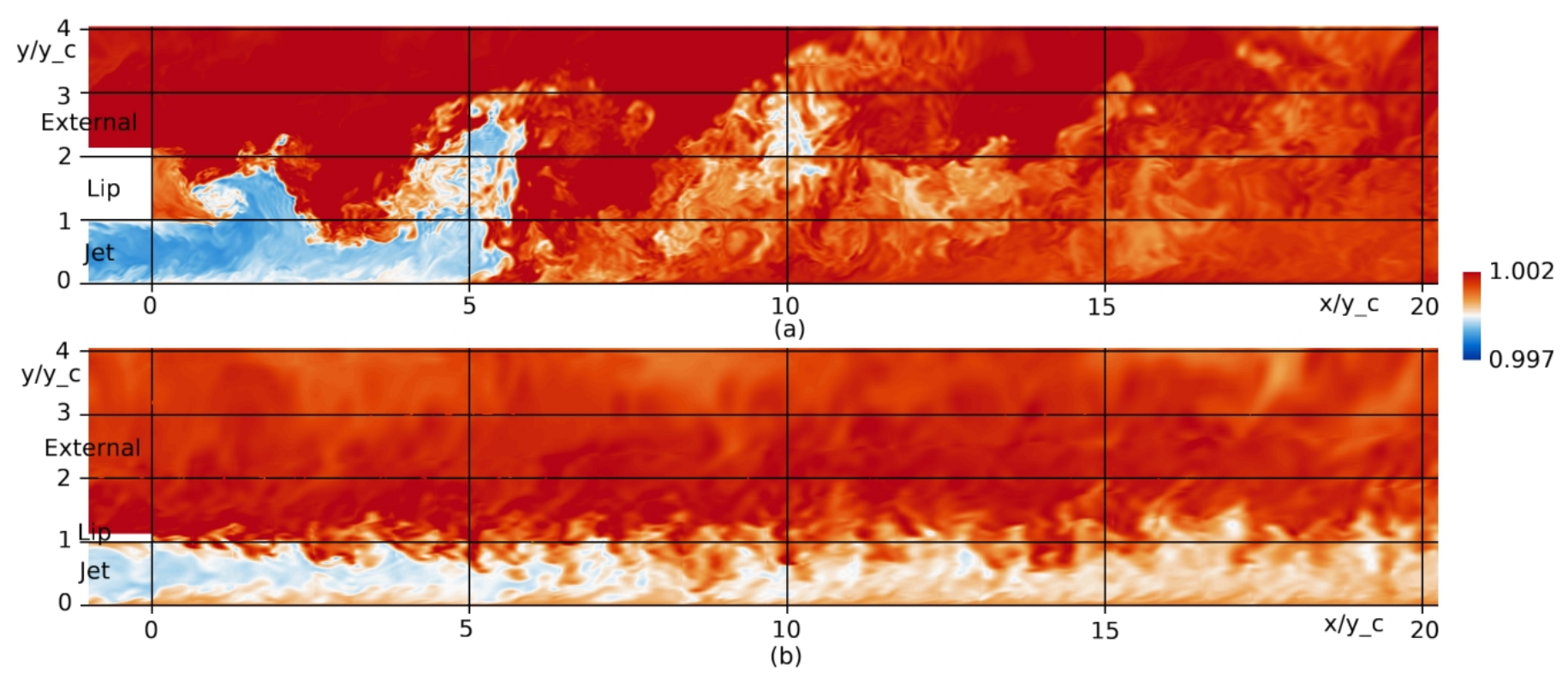

FIGURE 6: Instantaneous temperature contours in the $x-y$ plane for (a) Case A and (b) Case B.

Case A and B is interesting and warrants further investigation. Note that the numerical solutions are discontinuous at the element boundaries for DG schemes and show discontinuities in the mean pressure (Fig. 8) and wall shear stress (Fig. 9) distributions for $x>20 y_{c}$ due to lack of mesh resolution as discussed earlier. 


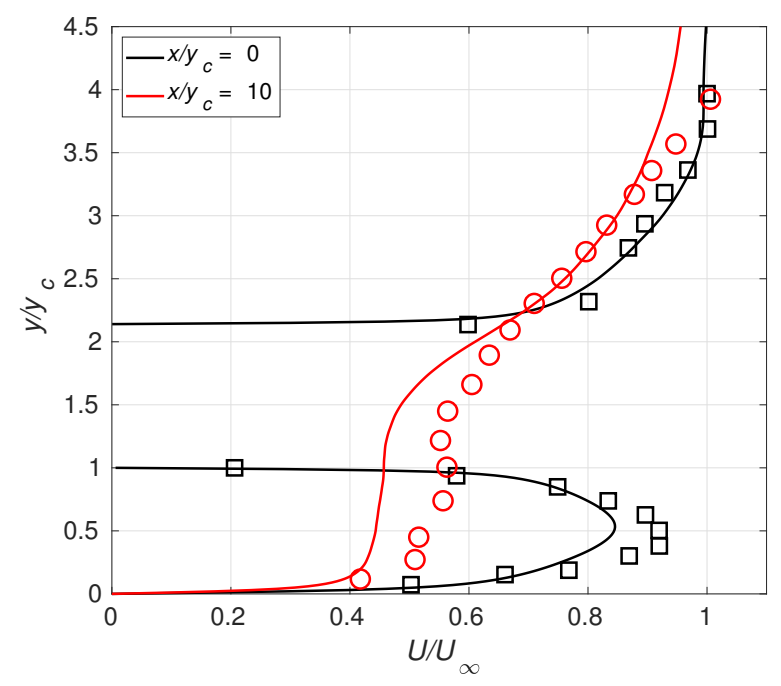

(a)

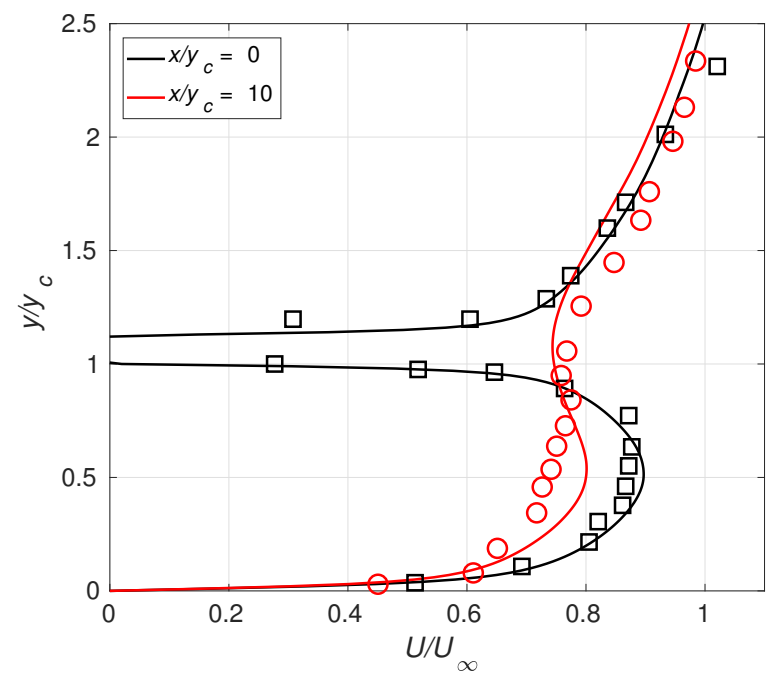

(b)

FIGURE 7: Comparison of mean streamwise velocity profiles with the experimental observations (the square and circle symbols represent $x=0$ and $10 y_{c}$, respectively) for (a) Case A, and (b) Case B.

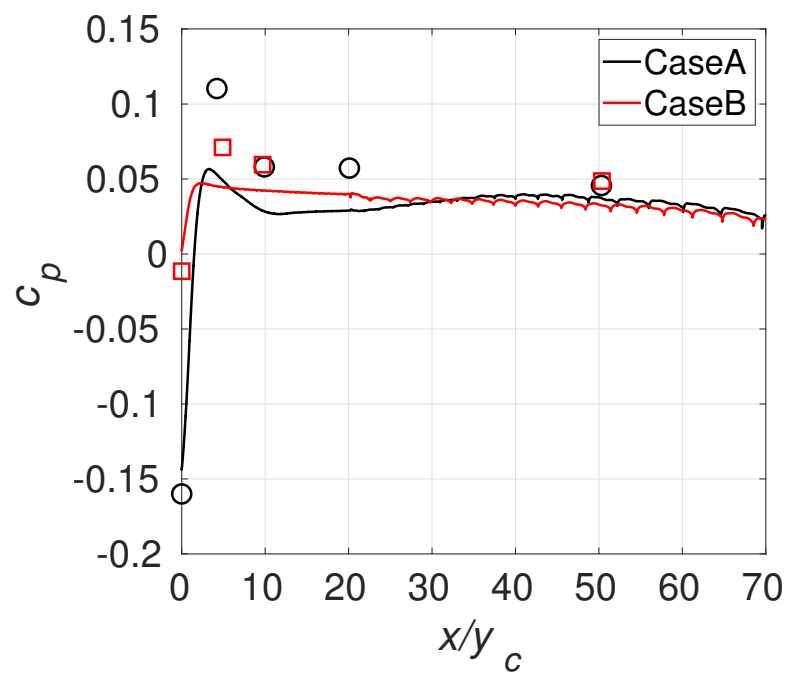

FIGURE 8: Comparison of the static pressure distribution at the base plate for Case A and Case B. The circles and squares represent experimental results for Case A and Case B, respectively.

\section{Turbulence Intensity}

The wall-normal profiles of the three components of the turbulence intensity are shown in Figs. 10. The present numerical predictions agree well with the experimental observations qualitatively and quantitative differences are most likely due to the

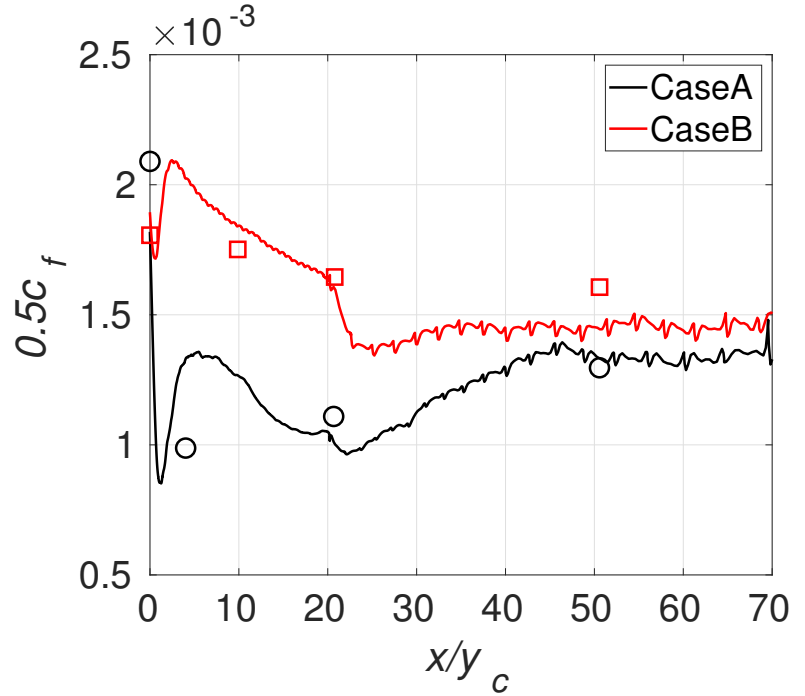

FIGURE 9: Comparison of the base plate wall shear stress for Case A and Case B. The circles and squares represent experimental results for Case A and Case B, respectively.

inflow turbulence assumptions.

For Case A, the streamwise (Fig. 10a) and wall-normal (Fig. 10b) components are predicted to be larger than the experimental observation for $x=4 y_{c}$ and $10 y_{c}$. The signatures of the jet and boundary-layer shear layer manifest themselves as two 


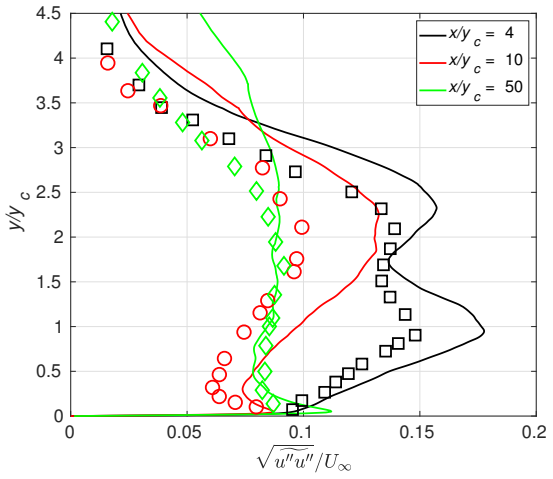

(a)

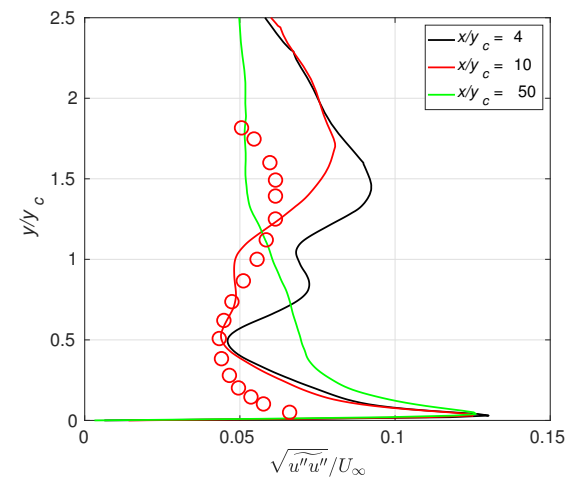

(d)

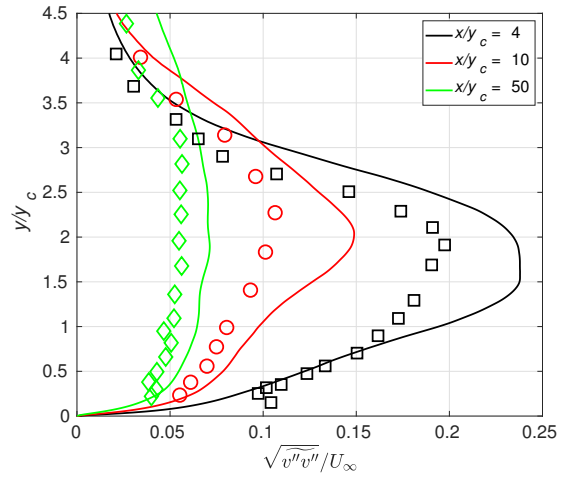

(b)

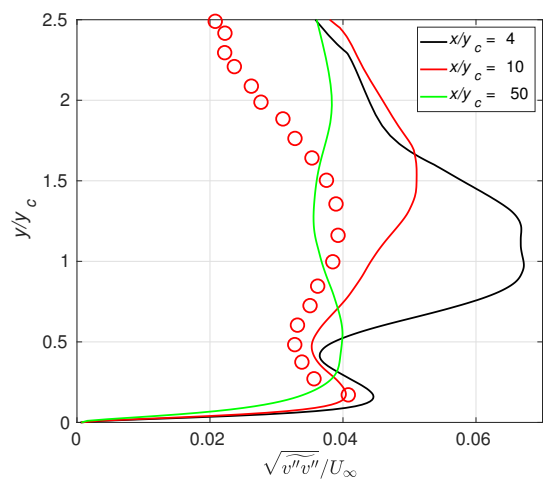

(e)

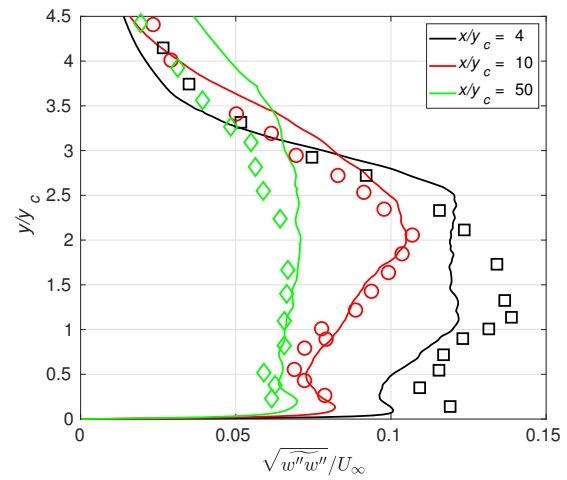

(c)

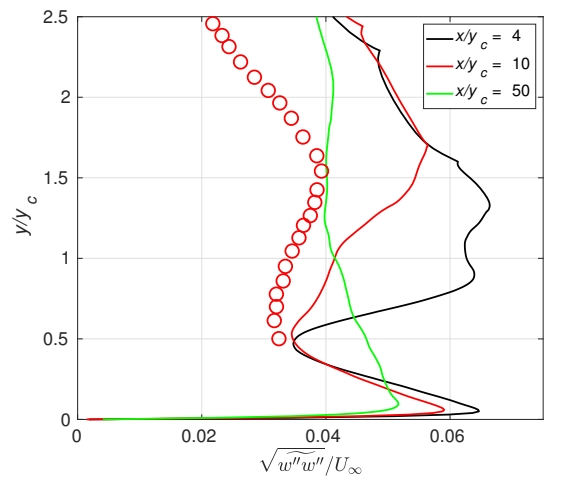

(f)

FIGURE 10: Turbulence intensity profiles for Case A $(a-c)$, and Case $B(d-f)$. Figures $(a, d),(b, e)$, and $(c, f)$ represent the streamwise, wall-normal, and spanwise turbulence intensity components, respectively. The square, circle, and diamond symbols represent the experimental observations at $x=4 y_{c}, 10 y_{c}$, and $50 y_{c}$, respectively.

peaks in the streamwise component at $x=4 y_{c}$. As the two shear layers merge with each other downstream, a single peak first appears $\left(x=10 y_{c}\right)$ at the outer part of the wall jet and then becomes almost constant with the wall-normal distance $\left(x=50 y_{c}\right)$. Another peak in the streamwise component also begins to appear near the wall at further downstream locations (Fig. 10a). The wall-normal component peaks in the middle of the wall-jet at $x=4 y_{c}$, and the magnitude of the peak decreases downstream while the peak location moves towards the outer part of the wall jet (Fig. 10b). The spanwise component is in better agreement with the experiment than the streamwise and wall-normal components. The spanwise component peaks near the wall and also at the middle of the jet for $x=4 y_{c}$, and at the outer part of the jet downstream (Fig. 10c).

For Case B, the streamwise turbulent intensity peaks near the wall (Fig. 10d) for all three locations. At $x=4 y_{c}$ the streamwise turbulent intensity decreases from the wall, and then increases at the jet and boundary-layer shear layers, and then decreases with the wall-normal distance. The two shear layers are less evident at the downstream locations, $x=10 y_{c}$ and $50 y_{c}$. At $x=$ $10 y_{c}$, the streamwise components first decrease from the wall, then increase to the outer part of the wall jet, and decrease again. At $x=50 y_{c}$, the streamwise component decreases with the wallnormal distance. The wall-normal component peaks near the wall and outer part of the wall jet (Fig. 10e). Similar to Case A, the outer peak of the wall-normal component decreases with streamwise distance. The spanwise component also shows two peaks, one near the wall and other at the outer part of the wall jet (Fig. 10f). The present simulations for Case B overpredict the turbulence intensities in the outer part of the wall jet compared to the experiment. This behavior is consistent with the observations of Naqavi et al. [16].

Note that the turbulence intensities are much smaller for Case B than Case A. The thick lip (Case A) results in higher turbulence due to the thicker lip wake. This also leads to better comparison with the experiment for Case A compared to Case B. 


\section{Reynolds Shear-Stress}

Wall-normal profiles of the Reynolds shear-stress $\left(\widetilde{u^{\prime \prime} v^{\prime \prime}}\right)$ are compared with the experimental observations in Fig. 11. For Case A, the shear-stress profile changes sign with the wallnormal distance at $x=4 y_{c}$ and $10 y_{c}$ due to the recirculation regions behind the lip (Fig. 5a). It is negative close to the wall, then increases to a positive value, drops again to a negative value, and then increases to zero at the outer part of the jet. The experimentally observed $\widetilde{u^{\prime \prime} v^{\prime \prime}}$ remains negative over the jet at $x=10 y_{c}$ for Case A; the present simulation also underestimates the peak negative value. For Case B, similar observations can be made, although the magnitude is much smaller compared to Case A. Similar to Ref. [16], the present simulation overpredicts the shearstress profile at the outer edge of the wall jet compared to the experimental observations for Case B.

\section{Mean Flowfield}

The two recirculating regions behind the lip that originate from the jet and boundary-layer shear layers as noted in the instantaneous flowfield visualizations for Case A (Fig. 5a) and Case B (Fig. 5b) can also be observed in the plots of the mean spanwise vorticity (Figs. 12a, 12b). The boundary-layer (or jet) shear layer results in a vortex that rotates in the clockwise (or anti-clockwise) direction. As the lip thickness is smaller for Case $\mathrm{B}$ compared to Case $\mathrm{A}$, the size of the recirculating regions are also smaller in the former case. These two recirculating vortices result in two peaks in the streamwise turbulence intensity component (Figs. 10a, 10d), and the oscillatory Reynolds shear stress (Figs. 11a, 11b) profiles. The anti-clockwise rotating vortex lifts up the jet core for Case A, resulting in a drop in the baseplate wall shear stress close to the wall-jet slot exit (Fig. 9). For Case B, no such lifting up of the jet core is observed. Most of the turbulence production is in the recirculating flow regions and production decreases downstream.

\section{Reynolds Stress Budget}

We next study the resolved Reynolds stress budgets for the two different configurations close to the jet slot to glean insights that can potentially inform RANS and/or LES models. The Reynolds stress $\left(\bar{\rho} u_{i}^{\prime \prime} u_{j}^{\prime \prime}\right)$ budget for compressible flow that has attained stationarity can be written as:

$$
0=M C_{i j}+P_{i j}+D_{i j}+P s_{i j}+P t_{i j}+T t_{i j}+V t_{i j}+M_{i j}
$$

where the right-hand-side terms in order are the mean convection, production, dissipation, pressure-strain, pressure transport, turbulent transport, viscous transport, and mass flux variation, respectively [42]. These terms are defined as:

$$
\begin{aligned}
& M C_{i j}=-\frac{\partial}{\partial x_{k}}\left(\tilde{u_{k}} \widetilde{\rho} \widetilde{u_{i}^{\prime \prime} u_{j}^{\prime \prime}}\right), \\
& P_{i j}=-\bar{\rho} \widetilde{u_{i}^{\prime \prime} u_{k}^{\prime \prime}} \frac{\partial \tilde{u}_{j}}{\partial x_{k}}-\bar{\rho} \widetilde{u_{j}^{\prime \prime} u_{k}^{\prime \prime}} \frac{\partial \tilde{u}_{i}}{\partial x_{k}}, \\
& D_{i j}=-\overline{\sigma_{i k}^{\prime} \frac{\partial u_{j}^{\prime}}{\partial x_{k}}}-\overline{\sigma_{j k}^{\prime} \frac{\partial u_{i}^{\prime}}{\partial x_{k}}}, P s_{i j}=\overline{p^{\prime}\left(\frac{\partial u_{j}^{\prime}}{\partial x_{i}}+\frac{\partial u_{i}^{\prime}}{\partial x_{j}}\right)}, \\
& P t_{i j}=-\frac{\partial \overline{p^{\prime} u_{i}^{\prime}}}{\partial x_{j}}-\frac{\partial \overline{p^{\prime} u_{j}^{\prime}}}{\partial x_{i}} \\
& T t_{i j}=-\frac{\partial \bar{\rho} \widetilde{u_{i}^{\prime \prime} u_{j}^{\prime \prime} u_{k}^{\prime \prime}}}{\partial x_{k}}, \\
& V t_{i j}=\frac{\partial\left(\overline{\sigma_{i k}^{\prime} u_{j}^{\prime}+\sigma_{j k}^{\prime} u_{i}^{\prime}}\right)}{\partial x_{k}}, \\
& M_{i j}=\overline{u_{i}^{\prime \prime}}\left(\frac{\partial \overline{\sigma_{j k}}}{\partial x_{k}}-\frac{\partial \bar{p}}{\partial x_{j}}\right)+\overline{u_{j}^{\prime \prime}}\left(\frac{\partial \overline{\sigma_{i k}}}{\partial x_{k}}-\frac{\partial \bar{p}}{\partial x_{i}}\right) \text {, } \\
& \sigma_{i j}=\mu\left(\frac{\partial u_{i}}{\partial x_{j}}+\frac{\partial u_{j}}{\partial x_{i}}-\frac{2}{3} \frac{\partial u_{k}}{\partial x_{k}} \delta_{i j}\right)
\end{aligned}
$$

where the overbar and tilde represent the Reynolds-averaged and Favre-averaged values, respectively, and / and // represent the deviations from the Reynolds and Favre averages, respectively. For the present simulations, the mass flux variation term, $M_{i j}$, is omitted from the figures since the compressibility effect $\left(\overline{u_{i}^{\prime \prime}} \approx 0\right)$ is negligible.

Turbulent Kinetic Energy Budget First, we study behavior of the budget terms for turbulent kinetic energy $\left(\bar{\rho} u_{i}^{\prime \prime} u_{i}^{\prime \prime}\right)$. Also, since $P s_{i i}$ is zero due to the flow being almost incompressible, and $V t_{i i}$ is zero except very close to the base plate, these terms are not shown in the plots (Figs. 13).

For Case A (Fig. 13a), $P_{i i}$ peaks at the two recirculating vortex regions from the jet and boundary-layer shear layers at $x=2 y_{c}$. For $1.0 y_{c}<y<2.2 y_{c}$, representing the lip wake region, the $P_{i i}$ and $M C_{i i}$ terms are mostly balanced by $T t_{i i}$. In the outer part of the lip wake regions, $T t_{i i}$ is balanced by $M C_{i i}$. At a downstream location, $x=10 y_{c} P_{i i}$ peaks close to the base plate and becomes non-zero close to the wall-jet edge. The $P_{i i}, P t_{i i}$, and $M C_{i i}$ terms are balanced by the $T t_{i i}$ and $D_{i i}$ terms close to the wall-jet edge, similar to the turbulent mixing layer budget [43]. The typical budget profile for wall-bounded flow [36] is recovered close to the base plate. In between these two regions, $T t_{i i}$ and $M C_{i i}$ are balanced by $D_{i i}$ and $P t_{i i}$.

For Case B (Fig 13b), there are both similarities as well as significant differences in the turbulent kinetic energy budget profiles when compared to Case A. At $x=2 y_{c}, P_{i i}$ also peaks close to the wall beside the two shear layers. At the lip wake region, 


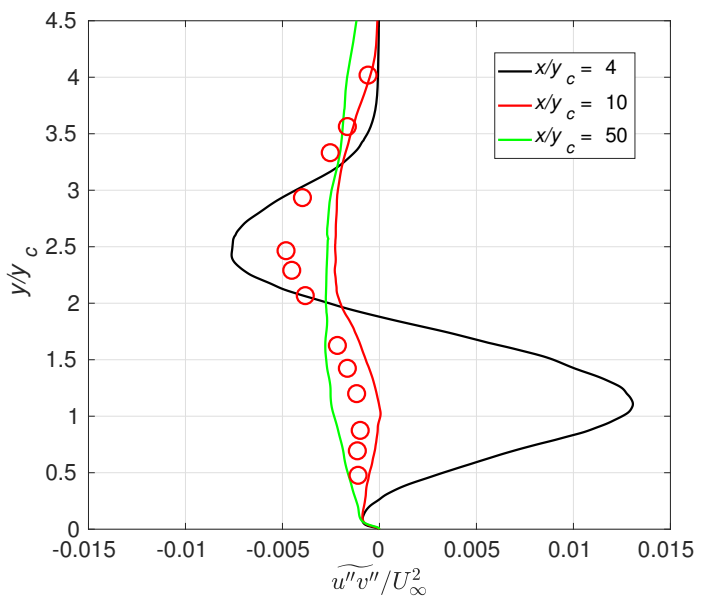

(a)

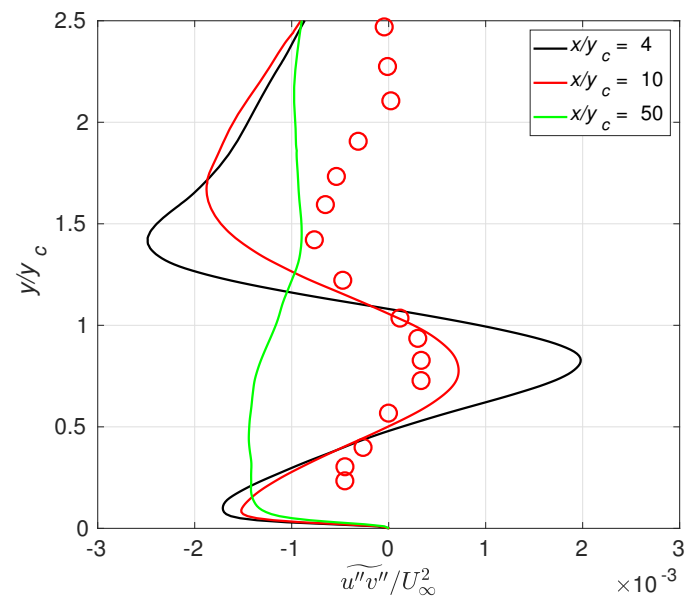

(b)

FIGURE 11: Shear-stress profiles for (a) Case A, and (b) Case B. The red circles represent experimental observations corresponding to the location $x=10 y_{c}$.

$0.8 y_{c}<y<1.2 y_{c}, M C_{i i}$ is stronger than $P_{i i}$, unlike for Case A. Close to the wall, $P_{i i}$ and $M C_{i i}$ are balanced by $D_{i i}$ and $T t_{i i}$. In the lip-wake region, similar to Case A, $M C_{i i}$ and $P_{i i}$ are balanced by the rest of the budget terms. At the outer part of the lip-wake region, $T t_{i i}$ is balanced by $M C_{i i}, P t_{i i}$ and $D_{i i}$. At $x=10 y_{c}$ the turbulent energy budget becomes similar to the wall-bounded flow. At the outer edge of the wall-jet, the turbulent energy budget terms are almost zero, unlike for Case A.

Reynolds Shear Stress Budget We next examine Reynolds shear-stress $\left(\bar{\rho} \widehat{u^{\prime \prime} v^{\prime \prime}}\right)$ budget profiles to understand the mixing mechanism in the wall-jet flows. For both Case A (Fig. 14a) and Case B (Fig. 14b), the shear stress budget profile is greatly influenced by the recirculating vortices from the jet and boundary-layer shear layers at $x=2 y_{c}$ location. At this location, $P_{12}$ is balanced by the rest of the budget terms. Shear-stress $P_{12}$ is positive in the jet shear layer location due to the anti-clockwise recirculating vortex, and negative in the boundary-layer shear layer location due to the clockwise recirculating vortex. Further downstream, $x=10 y_{c}$, the shear stress budget profile recovers the turbulent mixing layer budget profile [43]. At the outer edge of the wall jet, $P_{12}$ is negative and is balanced by the rest of the budget terms for Case A (Fig. 14a), although the shear-stress profile (Fig. 11a) is quite different that that in the turbulent mixing layer. For Case B, however, the budget terms are almost zero at the outer edge of the wall jet at $x=10 y_{c}$ (Fig. 14b). Near the wall, the $P s_{12}$ is balanced by the $P_{12}$ and $P t_{12}$ for both Case A and Case B, as is typical for wall-bounded flows [36] .

Note that the coarser mesh resolution used close to the outer edge of the wall jet compared to the wall results in discontinuities in the higher-order terms of the turbulent kinetic energy (Figs. 13) and Reynolds shear-stress budget (Figs. 14) profiles.

\section{CONCLUDING REMARKS}

We have performed wall-resolved LES using an entropystable DG formulation for two trailing-edge cooling slot configurations based on the experiments of Kacker \& Whitelaw [5] and have studied the effect of the lip geometry on the turbulent flow characteristics by varying the lip thickness from $0.126 y_{c}$ to $1.14 y_{c}$. A comparison of our results with the experimental observations demonstrate that the DG formulation can capture the turbulent mixing mechanism for such configurations.

Geometrical details upstream of the slot and the wind tunnel walls are not included in our simulations, and we have assumed fully-developed turbulent boundary layer and channel profiles at the inlet to the computational domain. Flow parameters for these inflow profiles are extrapolated by assuming zero-pressure gradient flow from the experimental observations at the slot exit. Our results qualitatively agree well with the experimental observations, and discrepancies between the experiments and our simulations may be attributed to the uncertainty in the turbulent inflow profiles, and the side-wall effects from the experimental tunnel. The present simulations reveal the importance of adequately documenting upstream turbulent inflow profiles and other boundary conditions in the experiments. Most turbulentinflow generation methods in the literature rely on the assumptions of fully-developed turbulent profiles that may not properly reflect experimental conditions. Our method for generating tur- 


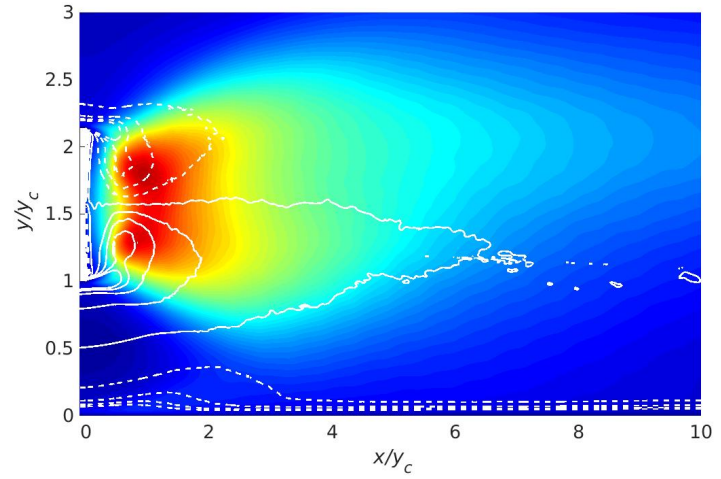

(a)

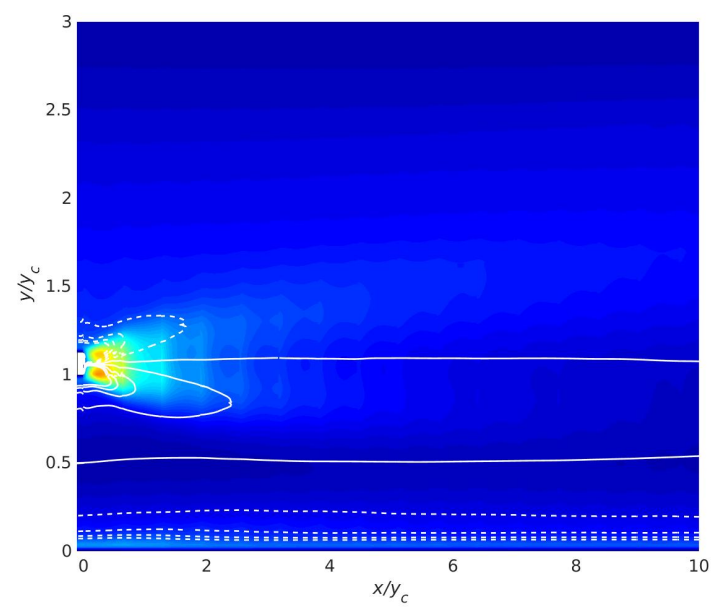

(b)

FIGURE 12: Contours of turbulent kinetic energy and mean spanwise vorticity for (a) Case A, and (b) Case B. The colormap represents turbulent kinetic energy, and the solid and dashed white lines represent anti-clockwise and clockwise mean spanwise vorticity, respectively.

bulent boundary layer profiles requires information regarding the mean streamwise gradients that again may not be available. A robust numerical technique that enforces arbitrary turbulent profiles at the inflow is needed to adequately perform inflow profile sensitivity studies.

The mixing characteristics of the wall-jet flow strongly depend on the lip thickness. Shear layers from the jet and the boundary layer result in two recirculating regions behind the lip. A thick lip results in larger recirculating regions compared to the thin lip. The shear layers form a vortex street, and the resulting vortex shedding portrays the turbulent mixing characteristics. The vortices grow more rapidly in the thick lip case compared to the thin lip case, and results in the jet core being lifted up close to the slot exit and higher unsteadiness in the jet. The different lip thicknesses lead to different mixing mechanisms that are manifested as differences in the energy budget terms.

\section{ACKNOWLEDGMENT}

Funding for this work was provided by the Advanced Air Transport Technology Project in the NASA Advanced Air Vehicles Program through a contract with Science and Technology Corporation. Additional funding was provided by the Transformational Tools and Technologies Project in the NASA Transformative Aeronautics Concepts Program. Computing resources were provided by the NASA Advanced Supercomputing facility at NASA Ames Research Center.

\section{REFERENCES}

[1] Whitelaw, J. H., 1967. An experimental investigation of the two-dimensional wall jet. Tech. Rep. C.P. 942, Aeronautical Research Council, Ministry of Technology, UK.

[2] Kacker, S. C., and Whitelaw, J. H., 1968. "Some properties of the two-dimensional turbulent wall jet in a moving stream". Journal of Applied Mechanics, 35, pp. 641-651.

[3] Kacker, S. C., and Whitelaw, J. H., 1968. "The effect of slot height and slot-turbulence intensity on the effectiveness of the uniform density, two-dimensional wall jet". Journal of Heat transfer, 90, pp. 469-475.

[4] Kacker, S. C., and Whitelaw, J. H., 1969. "An experimental investigation of the influence of slot-lip-thickness on the impervious-wall effectiveness of the uniform-density, two-dimensional wall jet". International Journal of Heat and Mass Transfer, 12, pp. 1201-1206.

[5] Kacker, S. C., and Whitelaw, J. H., 1971. "The turbulence characteristics of two-dimensional wall-jet and wall-wake flows". Journal of Applied Mechanics, 38, pp. 239-252.

[6] Martini, P., Schultz, A., Whitney, C. F., and Lutum, E., 2004. "Experimental and numerical investigation of trailing edge film cooling downstream of a slot with internal rib arrays". Journal of Turbomachinery, 126, pp. 229-236.

[7] Martini, P., Schultz, A., and Bauer, J. H., 2006. "Film cooling effectiveness and heat transfer on the trailing edge cutback of gas turbine airfoils with various internal cooling designs". Journal of Turbomachinery, 128, pp. 196-205.

[8] Menter, F. R., and Egorov, Y., 2005. "A scale-adaptive simulation modeling using two-equation models". AIAA Paper 2005-1095.

[9] Holloway, S. D., Leylek, J. H., and Buck, F. A., 2002. "Pressure-side bleed film cooling: Part 1, steady framework for experimental and computational results". ASME Turbo Expo Paper GT-2002-30471.

[10] Holloway, S. D., Leylek, J. H., and Buck, F. A., 2002. "Pressure-side bleed film cooling: Part 2, unsteady frame- 

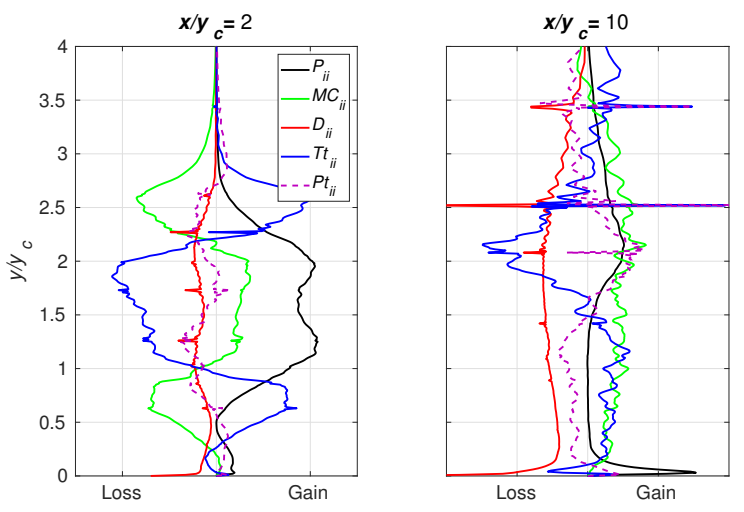

(a)
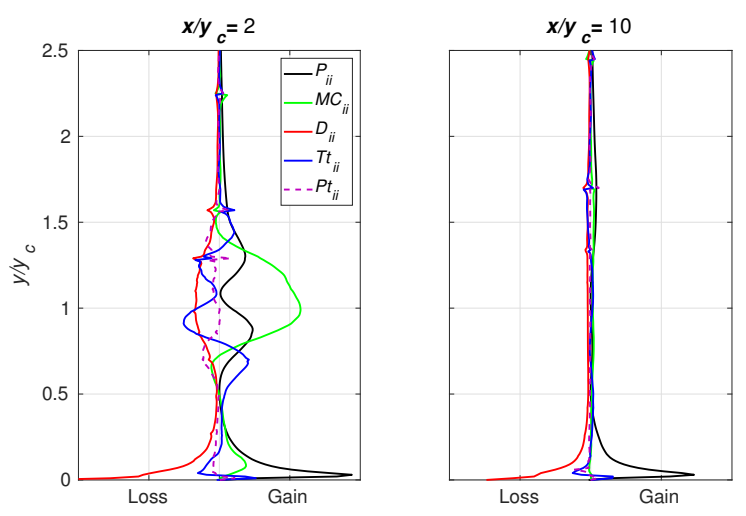

(b)

FIGURE 13: Turbulent kinetic energy budget profiles for (a) Case A, and (b) Case B at locations corresponding to $x / y_{c}=2$ and 10 . Note that the $P s_{i i}, V t_{i i}$, and $M_{i i}$ terms are omitted in the plots for clarity.
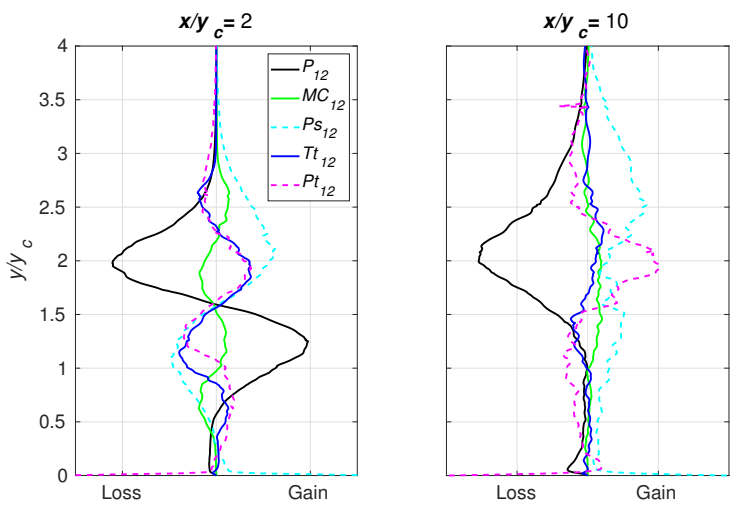

(a)
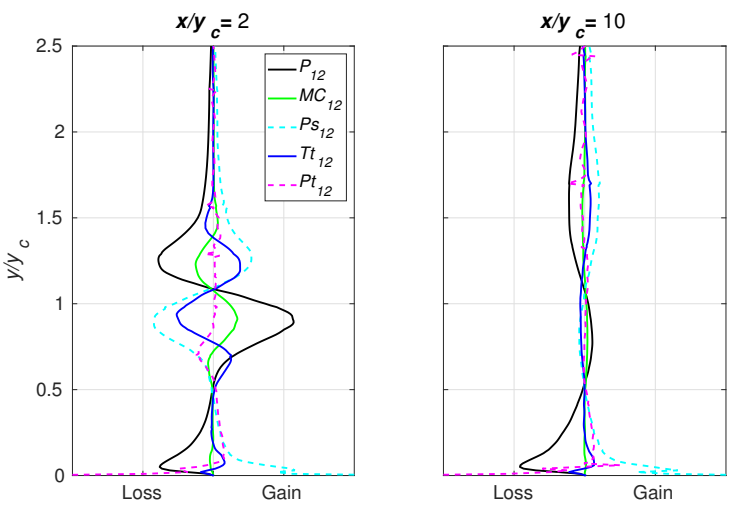

(b)

FIGURE 14: Reynolds shear stress budget profiles for (a) Case A, and (b) Case B at locations corresponding to $x / y_{c}=2$ and 10 . Note that the $D_{12}, V t_{12}$, and $M_{12}$ terms are omitted in the plots for clarity.

work for experimental and computational results". ASME Turbo Expo Paper GT-2002-30472.

[11] Medic, G., and Durbin, P. A., 2005. "Unsteady effects on trailing edge cooling". Journal of Heat Transfer, 127, pp. 388-392.

[12] Naqavi, I. Z., Tyacke, J. C., and Tucker, P. G., 2018. "Direct numerical simulation of a wall jet: flow physics". Journal of Fluid Mechanics, 852, pp. 507-542.

[13] Joo, J., and Durbin, P. A., 2009. "Simulation of turbine blade trailing edge cooling". Journal of Fluids Engineering, 131, p. 021102.

[14] Schneider, H., von Terzei, D., and Bauer, H. J., 2010. "Large-eddy simulations of trailing-edge cutback film cooling at low blowing ratio". International Journal of Heat and Fluid Flow, 31, pp. 767-775.

[15] Ivanova, E., and Laskowski, G. M., 2014. "LES and hybrid RANS/LES of a fundamental trailing edge slot". ASME Turbo Expo Paper GT2014-25906.

[16] Naqavi, I. Z., Tucker, P. G., and Liu, Y., 2014. "Largeeddy simulation of the interaction of wall jets with external stream". International Journal of Heat and Fluid Flow, 50, pp. 431-444.

[17] Sandberg, R. D., Tan, R., Weatheritt, J., Ooi, A., Haghiri, A., Michelassi, V., and Laskowski, G., 2018. "Applying machine learnt explicit algebraic stress and scalar flux models to a fundamental trailing edge slot". ASME Turbo Expo Paper GT-2018-75444.

[18] Bassi, F., Crivellini, A., Rebay, S., and Savini, M., 
2005. "Discontinuous Galerkin solution of the Reynoldsaveraged Navier-Stokes and $k-\omega$ turbulence model equations". Computers and Fluids, 34, pp. 507-540.

[19] Corsini, A., Rispoli, F., and Santoriello, A., 2005. "A variational multiscale higher-order finite-element formulation for turbomachinery flow computations". Computer Methods in Applied Mechanics and Engineering, 194, pp. 47974823.

[20] Cherednichenko, S., Frey, C., and Ashcroft, G., 2012. "On the application of the Discontinuous Galerkin method to turbomachinery flows". European Congress on Computational Methods in Applied Sciences and Engineering, pp. 2359-2375.

[21] Ghidoni, A., Colombo, A., and Rebay, S., 2013. "Simulation of the transitional flow in a low pressure gas turbine cascade with a high-order Discontinuous Galerkin method". Journal of Fluids Engineering, 135, p. 071101.

[22] Carton de Wiart, C., Hillewaert, K., and Geuzaine, P., 2012. "DNS of a low pressure turbine blade computed with a Discontinuous Galerkin method". ASME Turbo Expo Paper GT2012-68900.

[23] Hillewaert, K., Carton de Wiart, C., Verheylewegen, G., and Arts, T., 2014. "Assessment of a high-order Discontinuous-Galerkin method for the direct numerical simulation of transition at low Reynolds number in the T106C high-lift low pressure turbine cascade". ASME Turbo Expo Paper GT2014-26739.

[24] Carton de Wiart, C., Hillewaert, K., Lorriaux, E., and Verheylewegen, G., 2015. "Development of a Discontinuous Galerkin solver for high quality wall-resolved/modelled DNS and LES of practical turbomachinery flows on fully unstructured meshes". ASME Turbo Expo Paper GT201543428.

[25] Garai, A., Diosady, L. T., Murman, S. M., and Madavan, N. K., 2015. "DNS of flow in a low-pressure turbine cascade using a Discontinuous-Galerkin spectral-element method". ASME Turbo Expo Paper GT2015-42773.

[26] Garai, A., Diosady, L. T., Murman, S. M., and Madavan, N. K., 2016. "DNS of low-pressure turbine cascade flows with elevated inflow turbulence using a DiscontinuousGalerkin spectral element method". ASME Turbo Expo Paper GT2016-56700.

[27] Garai, A., Diosady, L. T., Murman, S. M., and Madavan, N. K., 2017. "Scale-resolving simulations of bypass transition in a high-pressure turbine cascade using a spectralelement Discontinuous-Galerkin method". Journal of Turbomachinery, 140(3), p. 031004.

[28] Garai, A., Diosady, L. T., Murman, S. M., and Madavan, N. K., 2018. "Scale-resolving simulations of low-pressure turbine cascades with wall roughness using a spectralelement method". ASME Turbo Expo Paper GT-201875982 .
[29] Diosady, L. T., and Murman, S. M., 2015. "Higher-order methods for compressible turbulent flows using entropy variables". AIAA Paper 2015-0294.

[30] Ismail, F., and Roe, P. L., 2009. "Affordable, entropyconsistent Euler flux functions II: entropy production at shocks". Journal of Computational Physics, 228, pp. 5410-5436.

[31] Bassi, F., and Rebay, S., 2000. Discontinuous Galerkin methods: theory, computation and applications. Springer, Berlin, Heidelberg, Germany, ch. GMRES Discontinuous Galerkin solution of the compressible Navier-Stokes equations, pp. 197-208.

[32] Diosady, L. T., and Murman, S. M., 2017. “Tensor-product preconditioners for higher-order space-time discontinuous galerkin methods". Journal of Computational Physics, 330, pp. 296-318.

[33] Carton de Wiart, C., Diosady, L. T., Garai, A., Burgess, N., Blonigan, P., Ekelschot, D., and Murman, S. M., 2018. "Design of a modular monolithic impicit solver for multiphysics applications". AIAA Paper 2018-1400.

[34] Hu, F. Q., Li, X. D., and Lin, D. K., 2008. “Absorbing boundary conditions for nonlinear Euler and Navier-Stokes equations based on the perfectly matched layer technique". Journal of Computational Physics, 227, pp. 4398-4424.

[35] Garai, A., Diosady, L. T., Murman, S. M., and Madavan, N. K., 2016. "Development of a perfectly matched layer technique for a Discontinuous-Galerkin spectral-element method". AIAA Paper 2016-1338.

[36] Pope, S. B., 2000. Turbulent flows. Cambridge University Press, Cambridge, UK.

[37] Schlichting, J., 1979. Boundary-layer theory. McGrawHill Book Company.

[38] Spalart, P. R., 1988. "Direct simulation of turbulent boundary layer up to $\operatorname{Re}_{\theta}=1410$ ". Journal of Fluid Mechanics, 187, pp. 61-98.

[39] Moser, R. D., Kim, J., and Mansour, N. N., 1999. “Direct numerical simulation of turbulent channel flow up to $R e_{\tau}=$ 590”. Physics of Fluids, 11(4), pp. 943-945.

[40] Murman, S. M., Diosady, L. T., and Garai, A., 2014. "Development of dynamic sub-grid models for variational multiscale methods". Center for Turbulence Research Proceedings of the Summer Program.

[41] Ekelschot, D., Ceze, M., Murman, S. M., and Garai, A., 2019. "Parallel high-order anisotropic meshing using discrete metric tensors". AIAA Paper 2019-1993.

[42] Gatski, T. B., 1997. New tools in turbulence modelling. Springer-Verlag, Berlin, Heidelberg, Germany, ch. Modelling compressibility effects on turbulence.

[43] Rogers, M. M., and Moser, R. D., 1994. "Direct simulation of a self-similar turbulent mixing layer". Physics of Fluids, 6, pp. 903-923. 